\title{
A finite volume scheme for anisotropic diffusion problems
}

\author{
Robert Eymard $^{\text {a }}$, T. Gallouët ${ }^{\text {b }}$, and Raphaèle Herbin ${ }^{\text {b }}$ \\ a Université de Marne-la-Vallée \\ E-mail:robert.eymard@univ-mlv.fr \\ b Université de Provence, 39 rue Joliot-Curie, 13453 Marseille \\ E-mail: gallouet, herbin@cmi.univ-mrs.fr
}

(Reçu le jour mois année, accepté après révision le jour mois année)

\begin{abstract}
A new finite volume for the discretization of anisotropic diffusion problems on general unstructured meshes in any space dimension is presented. The convergence of the approximate solution and its discrete gradient is proven. The efficiency of the scheme is shown through numerical examples. (C) 2002 Académie des sciences/Éditions scientifiques et médicales Elsevier SAS

Un schéma volumes finis pour les problèmes anisotropes sur des maillages non structurés

Résumé. On présente ici un nouveau schéma volumes finis pour la discrétisation des équations de diffusion anisotropes sur des maillages non structurés, pour toute dimension d'espace. On prouve la convergence de la solution approchée, ainsi que celle d'un gradient approché. La pertinence du schéma est illustrée par des résultats numériques. (c) 2002 Académie des sciences/Éditions scientifiques et médicales Elsevier SAS
\end{abstract}

\section{Introduction}

Anisotropic diffusion problems are encountered in various scientific fields: geosciences, biology, finance. Wellknown discretization methods are finite differences, finite volumes and finite elements. The mathematical study of convergence is quite wellknown in the case of isotropic diffusion for the three methods, and is the object of numerous works in the case of anisotropic diffusion; a thorough mathematical theory is known in the finite element theory. For various reasons, such as the ease of implementation, construction of simple Voronoï meshes and the coupling of equations of different kind, finite volumes are often chosen in actual applications. Our aim here is to construct a finite volume scheme for anisotropic problems and prove its convergence towards the solution of the continuous problem. We thus study the following problem: find an approximation of the variational weak solution $\bar{u} \in H_{0}^{1}(\Omega)$ to the following equation:

$$
-\operatorname{div}(\Lambda \nabla \bar{u})=f \text { in } \Omega,
$$

Note présentée par Philippe G. CIARLET

S0764-4442(00)0????-?/FLA

(C) 2002 Académie des sciences/Éditions scientifiques et médicales Elsevier SAS. Tous droits réservés. 


\section{R. Eymard, T. Gallouët, R. Herbin}

with homogeneous Dirichlet boundary conditions, where $\Omega$ is a polygonal open bounded connected subset of $\mathbb{R}^{d}, d \in \mathbb{N}^{*}$, and $f \in L^{2}(\Omega)$. The function $\Lambda$ is measurable from $\Omega$ to the set of symmetric $d \times d$ real matrices, the eigenvalues of which are included in $[\alpha(x), \beta(x)]$ where $\alpha, \beta \in L^{\infty}(\Omega)$ are such that $0<\alpha_{0} \leq \alpha(x) \leq \beta(x)$ for a.e. $x \in \Omega$.

The track which we follow here consists in building an approximate gradient, using the usual cell values of the discrete unknowns. Note that such an approximate gradient was already considered in [3]. However, in this latter work, the approximate gradient was constructed using the so-called Raviart-Thomas shape functions. Unfortunately, these functions have simple expressions only in the case of triangles and rectangles. Here, we shall develop a new approximate gradient which does not use these shape functions, and which is easy to compute on any admissible finite volume mesh.

\section{The finite volume scheme}

Let $\mathcal{T}=(\mathcal{M}, \mathcal{E}, \mathcal{P})$ be an admissible finite volume discretization of $\Omega$ in the sense of [2], Definition 9.1 page 762. An example of two neighbouring control volumes $K$ and $L$ of $\mathcal{N}$ is depicted in Figure 1 . The notations are identical to that of [2].

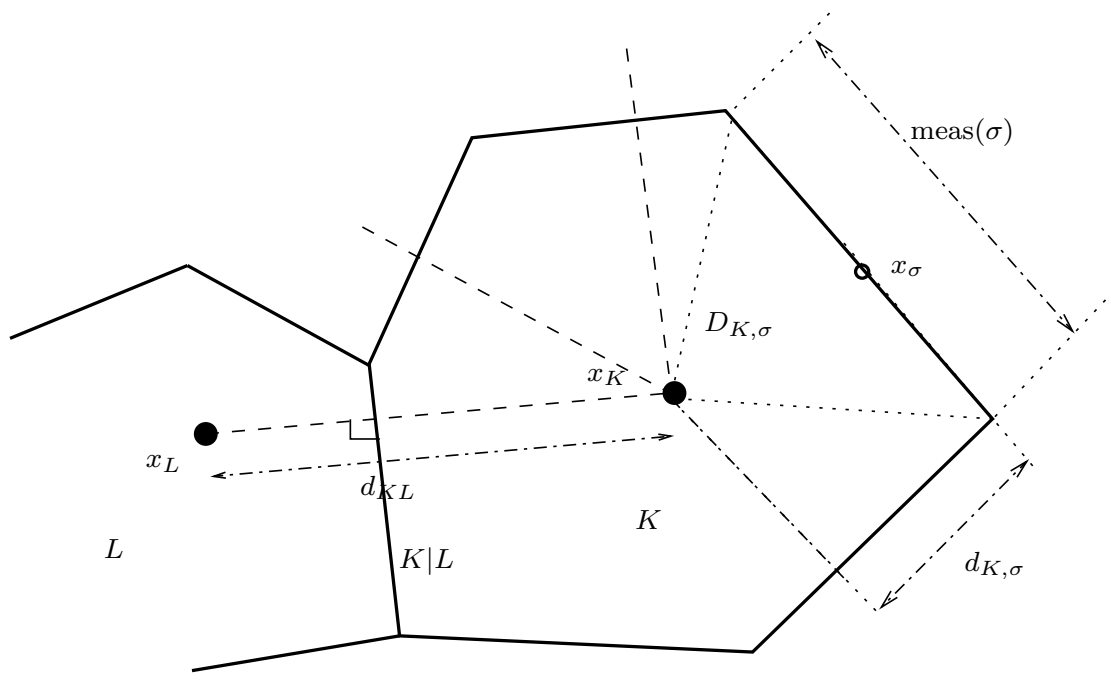

Figure 1: Notations for an admissible mesh

The size of the discretization is defined by:

$$
\operatorname{size}(\mathcal{T})=\sup \{\operatorname{diam}(K), K \in \mathcal{M}\} .
$$

The set of interior (resp. boundary) edges is denoted by $\mathcal{E}_{\text {int }}$ (resp. $\mathcal{E}_{\text {ext }}$ ). For any $\sigma \in \mathcal{E}_{\text {int }}, \sigma=K \mid L$ (resp. $\mathcal{E}_{\text {ext }}, \sigma \in \mathcal{E}_{K}$ ). For all $K \in \mathcal{M}$, we denote by $\mathcal{N}_{K}$ the subset of $\mathcal{M}$ of the neighbouring control volumes, and we denote by $\mathcal{E}_{K \text {,ext }}=\mathcal{E}_{K} \cap \mathcal{E}_{\text {ext }}$.

For all $K \in \mathcal{M}$ and $\sigma \in \mathcal{E}_{K}$, we define $D_{K, \sigma}=\left\{t x_{K}+(1-t) y, t \in(0,1), y \in \sigma\right\}$. For all $\sigma \in \mathcal{E}$, let $x_{\sigma}$ denote the center of gravity of $\sigma$. We shall measure the regularity of the mesh through the function $\operatorname{regul}(\mathcal{T})$ defined by

$$
\operatorname{regul}(\mathcal{T})=\inf \left\{\frac{d_{K, \sigma}}{d\left(x_{\sigma}, x_{K}\right)}, K \in \mathcal{M}, \sigma \in \mathcal{E}_{K}\right\} .
$$

We denote by $H_{\mathcal{T}}(\Omega) \subset L^{2}(\Omega)$ the space of piecewise constant functions on the control volumes. For all $w \in H_{\mathcal{T}}(\Omega)$ and for all $K \in \mathcal{M}$, we denote by $w_{K}$ the constant value of $w$ in $K$. For any function 


\section{A finite volume scheme for anisotropic diffusion problems}

$\varphi \in C_{c}^{\infty}(\Omega)$, we denote by $P_{\mathcal{M}} \varphi \in H_{\mathcal{T}}(\Omega)$ the piecewise function equal to $\varphi\left(x_{K}\right)$ on cell $K$. For $(v, w) \in\left(H_{\mathcal{T}}(\Omega)\right)^{2}$, we denote by:

$$
\begin{aligned}
{[v, w]_{\mathcal{T}, \alpha}=} & \sum_{K \mid L \in \mathcal{E}_{\text {int }}} \frac{\operatorname{meas}(K \mid L)}{d_{K L}} \frac{\left(v_{L}-v_{K}\right)\left(w_{L}-w_{K}\right)}{\operatorname{meas}\left(D_{K, K \mid L} \cup D_{K, K \mid L}\right)} \int_{D_{K, K \mid L} \cup D_{K, K \mid L}} \alpha(x) d x \\
& +\sum_{\sigma \in \mathcal{E}_{\text {ext }}} \frac{m_{\sigma}}{d_{K, \sigma}} \frac{v_{K} w_{K}}{\operatorname{meas}\left(D_{K, \sigma}\right)} \int_{D_{K, \sigma}} \alpha(x) d x .
\end{aligned}
$$

We define a norm in $H_{\mathcal{T}}(\Omega)$ by $\|w\|_{\mathcal{T}}=\left([w, w]_{\mathcal{T}, 1}\right)^{1 / 2}$ (where 1 denotes the constant function equal to 1$)$. We define the discrete gradient $\nabla_{\mathcal{T}}: H_{\mathcal{T}}(\Omega) \rightarrow H_{\mathcal{T}}(\Omega)^{d}$, by:

$$
\begin{aligned}
& \operatorname{meas}(K) \nabla_{\mathcal{T}} w_{K}=\sum_{L \in \mathcal{N}_{K}} \frac{\operatorname{meas}(K \mid L)}{d_{K L}}\left(x_{K \mid L}-x_{K}\right)\left(w_{L}-w_{K}\right)-\sum_{\sigma \in \mathcal{E}_{K, \text { ext }}} \frac{\operatorname{meas}(\sigma)}{d_{K, \sigma}}\left(x_{\sigma}-x_{K}\right) w_{K}, \\
& \forall K \in \mathcal{M} .
\end{aligned}
$$

We consider the finite volume scheme:

$$
\left\{\begin{array}{l}
u_{\mathcal{T}} \in H_{\mathcal{T}}(\Omega), \\
\int_{\Omega}(\Lambda(x)-\alpha(x) I d) \nabla_{\mathcal{T}} u_{\mathcal{T}}(x) \cdot \nabla_{\mathcal{T}} v(x) d x+\left[u_{\mathcal{T}}, v\right]_{\mathcal{T}, \alpha}=\int_{\Omega} f(x) v(x) d x, \forall v \in H_{\mathcal{T}}(\Omega) .
\end{array}\right.
$$

One may prove that there exists a unique solution $u_{\mathcal{T}}$ to (3), thanks to the following discrete $H^{1}$ estimate:

$$
\alpha_{0}\left\|u_{\mathcal{T}}\right\|_{\mathcal{T}} \leq \operatorname{diam}(\Omega)\|f\|_{L^{2}(\Omega)^{d}} .
$$

\section{Convergence results}

THEOREM 3.1. - [Convergence of the finite volume scheme] Let $\mathcal{T}$ be an admissible discretization of $\Omega$. Let $u_{\mathcal{T}} \in H_{\mathcal{T}}(\Omega)$ be the solution to (3). Then $u_{\mathcal{T}}$ converges in $L^{2}(\Omega)$ to the weak solution $\bar{u}$ to the problem (1), and the approximate gradient $\nabla_{\mathcal{T}} u_{\mathcal{T}}$ converges in $L^{2}(\Omega)^{d}$ to $\nabla \bar{u}$, as $\operatorname{size}(\mathcal{T}) \rightarrow 0$ provided that that there exists $\rho>0$ with $\operatorname{regul}(\mathcal{T}) \geq \rho$.

Remark 1. - Note that the present convergence result also gives the strong convergence of the present discrete gradient for the classical finite volume scheme in the isotropic case, which is given by (3) when taking $\Lambda=\alpha I d$.

Sketch of proof of Theorem 3.1: From (4), we may extract from any sequence of approximate solutions a subsequence which converges to some $u \in H_{0}^{1}(\Omega)$. We shall show below that $u=\bar{u}$, and therefore, $u$ is unique. Hence we may consider any family of discretizations $\mathcal{T}$ with $\operatorname{regul}(\mathcal{T}) \geq \rho$, and $\operatorname{size}(\mathcal{T}) \rightarrow 0$.

Let $\varphi \in C_{c}^{\infty}(\Omega)$, set $v=P_{\mathcal{T}} \varphi$ in (3). The keypoints of the proof of convergence of $u_{\mathcal{T}}$ and $\nabla_{\mathcal{T}} u_{\mathcal{T}}$ are the following:

1. $\left[u_{\mathcal{T}}, P_{\mathcal{T}} \varphi\right]_{\mathcal{T}, \alpha} \rightarrow \int_{\Omega} \alpha(x) \nabla u(x) \cdot \nabla \varphi(x) d x$,

2. $\nabla \mathcal{T} u_{\mathcal{T}}$ tends to $\nabla u$ weakly in $L^{2}(\Omega)^{d}$,

3. $\nabla_{\mathcal{T}} P_{\mathcal{T}} \varphi$ tends to $\nabla \varphi$ in $L^{2}(\Omega)^{d}$.

Item 1 is developped in [2], [1]. Item 2 may be shown by noting that, thanks to the orthogonality of $x_{K} x_{L}$ and $K \mid L$, we have

$\sum_{K \mid L \in \mathcal{E}_{\mathrm{int}}}\left(u_{L}-u_{K}\right)\left[\mathbf{n}_{K, L} \int_{K \mid L} \varphi(x) d \gamma(x)-\frac{\operatorname{meas}(K \mid L)}{d_{K L}}\left(\left(x_{K \mid L}-x_{K}\right) \varphi\left(x_{K}\right)+\left(x_{L}-x_{K \mid L}\right) \varphi\left(x_{L}\right)\right)\right] \rightarrow 0$ 


\section{R. Eymard, T. Gallouët, R. Herbin}

which leads to

$$
\int_{\Omega} P_{\mathcal{T}} \varphi(x) \nabla_{\mathcal{T}} u_{\mathcal{T}}(x) d x+\int_{\Omega} u_{\mathcal{T}}(x) \nabla \varphi(x) d x \rightarrow 0 .
$$

Item 3 is a consequence of the following geometric property: for all $i, j=1, \ldots, d$,

$\int_{K} \operatorname{div}\left(x^{(i)} \mathbf{e}^{(j)}\right) d x=\delta_{i j} \operatorname{meas}(K)=\sum_{\sigma \in \mathcal{E}_{K}} \operatorname{meas}(\sigma) x_{\sigma}^{(i)} \mathbf{n}_{K, \sigma} \cdot \mathbf{e}^{(j)}$, obtained thanks to the choice of $x_{\sigma}$ as the center of gravity of $\sigma$. One then concludes to the convergence of $u_{\mathcal{T}}$ thanks to the fact that $\int_{\Omega}(\Lambda(x)-\alpha(x) I d) \nabla_{\mathcal{T}} u(x) \cdot \nabla_{\mathcal{T}} P_{\mathcal{T}} \varphi(x) d x$ tends to $\int_{\Omega}(\Lambda(x)-\alpha(x) I d) \nabla u(x) \cdot \nabla \varphi(x) d x$, and thus $u=\bar{u}$.

In order to prove the convergence of $\nabla_{\mathcal{T}} u_{\mathcal{T}}$, we consider a regular function $\psi$, and write the following inequality:

$$
\left\|\nabla_{\mathcal{T}} u-\nabla u\right\|_{L^{2}(\Omega)^{d}} \leq\left\|\nabla_{\mathcal{T}} u-\nabla_{\mathcal{T}} P_{\mathcal{T}} \psi\right\|_{L^{2}(\Omega)^{d}}+\left\|\nabla_{\mathcal{T}} P_{\mathcal{T}} \psi-\nabla \psi\right\|_{L^{2}(\Omega)^{d}}+\|\nabla \psi-\nabla u\|_{L^{2}(\Omega)^{d}} .
$$

Now, it may be shown that $\left[u_{\mathcal{T}}, u_{\mathcal{T}}\right]_{\mathcal{T}, \alpha}$ tends to $\int_{\Omega} \alpha(x) \nabla u(x)^{2} d x$, thanks to "lim inf" considerations [4], using weak convergence properties. Hence we get that $\lim \sup _{\operatorname{size}(\mathcal{T}) \rightarrow 0}\left\|\nabla_{\mathcal{T}} u-\nabla_{\mathcal{T}} P_{\mathcal{T}} \psi\right\|_{L^{2}(\Omega)^{d}} \leq$ $C\|\nabla \psi-\nabla u\|_{L^{2}(\Omega)^{d}}$. Using item 3, one has $\lim \sup _{\operatorname{size}(\mathcal{T}) \rightarrow 0}\left\|\nabla_{\mathcal{T}} u_{\mathcal{T}}-\nabla u\right\|_{L^{2}(\Omega)^{d}} \leq(C+1) \| \nabla \psi-$ $\nabla u \|_{L^{2}(\Omega)^{d}}$. Letting $\psi$ tend to $u$ in $H_{0}^{1}(\Omega)$, we conclude.

\section{Numerical results}

We plot in the figure on the right the error between exact and approximate solution versus the parameter $\alpha$ for problem (1) with $\Omega=(0,1) \times(0,1), \Lambda(x)=\left(\begin{array}{cc}1.5 & .5 \\ .5 & 1.5\end{array}\right)$, and $\bar{u}(x)=x^{(1)}(1-$ $\left.x^{(1)}\right) x^{(2)}\left(1-x^{(2)}\right)$, using a uniform square $25 \times 25$ grid. We observe that a minimum is attained for a constant value of $\alpha(x)$ close to 2 , that is the largest eigenvalue of $\Lambda(x)$. However, the above proof of convergence is only valid for $\alpha \leq 1$, that is the smallest eigenvalue of $\Lambda(x)$. Hence one should aim at proving the convergence for larger values of $\alpha$. For this same problem, the order of convergence of the method with respect to the size of the mesh is close to 2 for the solution for both rectangles and triangles, and 1.5 (resp. 1) for its gradient in the case of rectangles (resp. triangles).

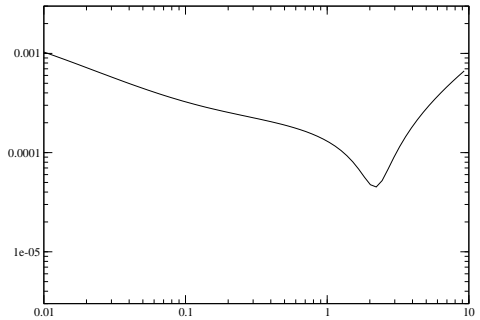

$L^{2}$ norm of the error as a function of $\alpha$

\section{References}

[1] R. Eymard and T. Gallouët, H-convergence and numerical schemes for elliptic equations SIAM Journal on Numerical Analysis, 41, 2, 539-562, 2003.

[2] R. Eymard, T. Gallouët and R. Herbin, Finite Volume Methods, Handbook of Numerical Analysis, Vol. VII, 713-1020. Edited by P.G. Ciarlet and J.L. Lions (North Holland), 2000.

[3] R. Eymard, T. Gallouët and R. Herbin, Finite volume approximation of elliptic problems and convergence of an approximate gradient", Appl. Num. Math., 37, 1-2, 31-53, 2001.

[4] R. Herbin and E. Marchand, Finite volume approximation of a class of variational inequalities, IMA J. Numer. Anal., 21, 2, 553-585, 2001. 